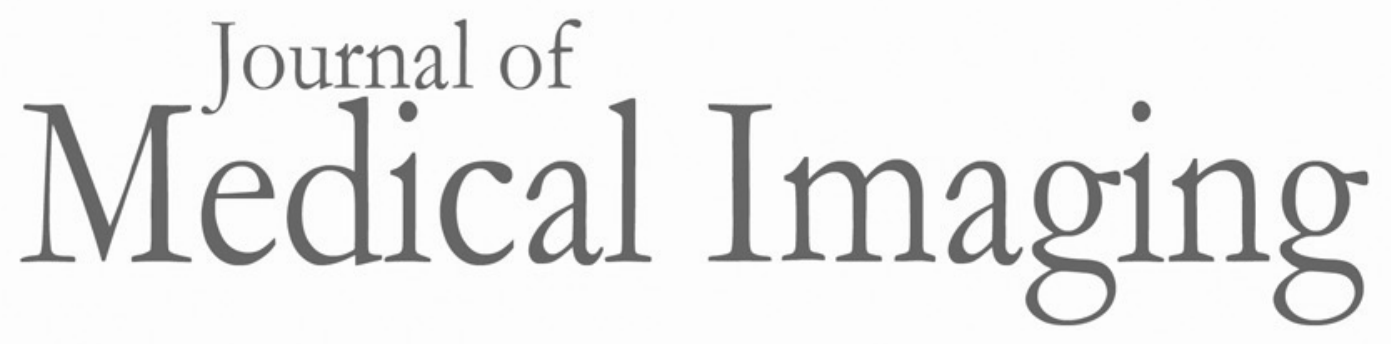

Medicallmaging.SPIEDigitalLibrary.org

\title{
Special Section on 3D Printing in Medical Imaging
}

Ehsan Samei

Joseph Lo 


\title{
Special Section on 3D Printing in Medical Imaging
}

\author{
Ehsan Samei, PhD \\ Joseph Lo, PhD \\ Carl E. Ravin Advanced Imaging Laboratories \\ Department of Radiology \\ Duke University School of Medicine \\ Durham, North Carolina, USA
}

What is 3D printing? Rapid prototyping, additive manufacturing, or $3 \mathrm{D}$ printing all refer to technologies that fabricate objects quickly and inexpensively. These processes can take a 3D computer design and transform it into physical form by depositing material layer by layer. Originally intended for preliminary prototypes, the latest 3D printing technology can produce end products that deliver quality and specifications even better than traditional manufacturing processes. There are many different 3D printing technologies, including fused filament fabrication, stereolithography, polymer inkjet, paper inkjet, and many more. These technologies provide an extraordinary range and flexibility for transforming ideas into physical reality.

Why 3D printing in medical imaging? Given the amazing capabilities of $3 D$ printing, it is no surprise that the medical imaging field has embraced these technologies. Already many hospitals are adopting 3D printing to design patientspecific surgical plans and implants, resulting in measurable clinical impact in terms of improved quality and safety. Even more exciting possibilities have emerged in medical imaging research. At the 2017 SPIE Medical Imaging conference, a workshop entitled "3D Printing in Medical Imaging" showcased some of these new research topics, including anthropomorphic breast phantoms for varying imaging modalities, specialized imaging instruments, and even living tissue samples. 3D printing can translate virtual designs and medical imaging data alike into the real world.

In this issue: this special section aims to highlight some of the applications of 3D printing in medical imaging. In particular, the issue has five informative papers. Gerbl et al. in their paper titled "Characterization of office laser printers for 3-D printing of soft tissue CT phantoms" showcase how commonplace office hardware can be repurposed to create realistic imaging phantoms.

Shepard et al. in "Initial evaluation of three-dimensionally printed patient-specific coronary phantoms for CT-FFR software validation" use polymer inkjetting to create functional phantoms that mimic the physiological flow and pressure conditions of actual patients.

In "Three-dimensionally-printed anthropomorphic physical phantom for mammography and digital breast tomosynthesis with custom materials, lesions, and uniform quality control region," Rossman et al. "hack" polymer inkjetting to create bespoke breast phantoms with characteristics that cannot be achieved by any commercial phantom making techniques.

Nouls et al., in their paper titled "Three-dimensional printing applications in small-animal MRI," draw upon both filament fabrication and selective laser sintering to create a myriad of

(C) 2019 Society of Photo-Optical Instrumentation Engineers (SPIE)
MR-compatible parts and instruments customized for five separate preclinical studies.

Finally, Samei et al. in their paper titled "Design and fabrication of heterogeneous lung nodule phantoms for assessing the accuracy and variability of measured texture radiomics features in CT" use polymer inkjetting to create lesions that can provide ground truth for radiomics measurements. This demonstrates the potential for 3D printing to address issues in a way that cannot be done with in vivo patient data.

What is next? Beyond the topics above, 3D printing covers a wide range of topics. They include advances in technology and material science related to printing:

- New 3D printing technologies

- Comparison of 3D printing technologies

- Resolution improvements and voxelized printing

- Image processing for and from 3D printed objects (eg, segmentation, rendering)

- Advanced computation for and from 3D printed objects (eg, virtual reality, deep learning)

- Manufacturing evaluation and improvements (eg, pertaining consistency, repeatability, price)

- Tissue-equivalent materials for 3D printing

- Printing with multiple materials

- Bio-compatible materials.

The technology as applied to basic and translational research can cover a wide range of areas:

- Bioprinting, tissue engineering, regenerative medicine

- Instrumentation for small animal imaging

- Phantoms for diagnostic imaging

- Phantoms for dosimetry

- Phantoms for radiation oncology

- Phantoms for nuclear medicine

- Anthropomorphic or patient-specific phantoms

- Contrast imaging

- Radiomics

- Instrumentation for imaging or therapy.

Finally, in clinical practice, 3D printing objects are paving their way into multiple uses:

- Surgical planning

- Patient-specific implants

- Regulatory constraints 
- Use of 3D objects for virtual clinical trials

- Needed printing quality for clinically-representative predictions

- The integrated use of 3D printed objects for clinical optimization.

We encourage the readers of this special issue to ponder and tackle these relevant topics. We hope papers corresponding to these topics will be published in future issues of JMI. And the results will hopefully lead to a measurable impact on the reach of medical imaging to advance medicine and associated techniques.

Ehsan Samei, PhD, is a tenured professor at Duke University, where he serves as the director of the Duke Medical Physics Graduate
Program and Clinical Imaging Physics. His interests include clinicallyrelevant metrology of imaging quality and safety for optimum interpretive and quantitative performance. He strives to bridge the gap between scientific scholarship and clinical practice by meaningful realization of translational research and by actualization of clinical processes that are informed by scientific evidence.

Joseph Lo, PhD, is a professor and associate vice chair for research at the Department of Radiology, Duke University School of Medicine. $\mathrm{He}$ is also director of the Carl E. Ravin Advanced Imaging Laboratories (RAI Labs), an interdisciplinary group composed of six core faculty members from Duke Radiology and over 40 other faculty, staff, and students. RAI Labs members investigate imaging technology and data science for x-ray and other modalities, and work closely with radiologists to facilitate clinical translation. Dr. Lo's research includes creating virtual and physical phantoms based on patient anatomy, as well as applying machine learning to improve breast and CT imaging. 